\title{
Der kolumbianische Kaffee
}

Hans J. Tanner

Kolumbien ist neben Brasilien der größte Kaffeeproduzent der Erde. Im Kaffeejahr 1965/66 (das Kaffeejahr beginnt am 1. Oktober) betrug die Produktion Brasiliens $2100000 \mathrm{t}$ oder $44,4 \%$ der Welterzeugung; Kolumbien produzierte $480000 \mathrm{t}$ $(10,15 \%)$, während das nächstwichtigste Land, die Elfenbeinküste, mit 276000 t $(5,83 \%)$ in den Statistiken figuriert (1).

Kolumbien ist aber bei weitem der wichtigste Produzent von gewaschenem Kaffee. Der brasilianische Kaffee wird ungewaschen exportiert.

In der unter dem Weltkaffeeabkommen von 1962 autorisierten Exportquotenliste werden die Kaffeeproduktionsländer in Gruppen eingeteilt: 1. in die Exportländer von Robustakaffee und 2. in die Exporteure von Arabicakaffee, die in drei Untergruppen zerfallen: a) in Exporteure von gewaschenem mildem Kaffee aus Kolumbien (arabigo suave colombiano): b) anderem mildem gewaschenem Kaffee (otros arabigos suaves) und c) ungewaschenen Arabicas (arabigos no lavados). Von der für das Kaffeejahr 1966/67 bewilligten Gesamtweltausfuhr von 41,7 Millionen Sack (zu $60 \mathrm{~kg}$ ) entfallen 9759856 Sack auf die Gruppe 1a, 6240512 auf die Gruppe 2a (davon 5387100 auf Kolumbien), 8491810 Sack auf die Gruppe 2b und 17207822 Sack auf die Gruppe 2c (wovon auf Brasilien 16130972 Sack).

In der Gruppe 2a ist neben Kolumbien und Kenia seit Februar 1967 auch Tansania eingegliedert (9).

\section{Die volkswirtschaftliche Bedeutung des} kolumbianischen Kaffees

Seit Jahren ist der Kaffee das weitaus wichtigste Ausfuhrprodukt Kolumbiens. In den vierziger und fünfziger Jahren waren zeitweise $90 \%$ der Deviseneinnahmen des Landes auf die Kaffee-Exporte zurückzuführen. Schwankungen des Kaffeepreises auf dem Weltmarkt machen sich deshalb im kolumbianischen Nationaleinkommen sofort stark bemerkbar. Es gilt die Faustregel, daß ein Absinken des Kaffeepreises um 1 US-Cent je Pfund im Jahresdurchschnitt das Land 7 Millionen US-Dollar kostet. 1954 hatte Kolumbien bei Rekordpreisen von über 90 Cent aus dem Kaffee 550 Millionen US-Dollar gelöst, 1965 bei Preisen von 41 bis 42 Cent betrugen die Deviseneinnahmen aus dem Kaf-
fee-Export trotz einer um etwa $10 \%$ größeren Ausfuhr nur noch 344 Millionen US-Dollar.

Trotz allen Bemühungen der letzten Jahre, durch eine verstärkte Industrialisierung und die Intensivierung anderer Zweige der tropischen Landwirtschaft die kolumbianische Volkswirtschaft auf eine breitere Basis zu stellen, ist der Anteil der Kaffeeproduktion seit 1950 um weniger als $2 \%$ zurückgegangen, und der Kaffee ist bei weitem das wichtigste Einzelprodukt der kolumbianischen Volkswirtschaft geblieben.

\begin{tabular}{|c|c|c|}
\hline \multicolumn{2}{|c|}{ Bruttonationalprodukt } & davon Kaffee \\
\hline 1950 & 14689 & 1430 \\
\hline 1955 & 18976 & 1573 \\
\hline 1960 & 23042 & 1951 \\
\hline 1964 & 27812 & 2178 \\
\hline
\end{tabular}

(in Millionen Pesos, zu konstanten Preisen des Jahres 1958)

\section{Die Entwicklung des Kaffeeanbaus in Kolumbien}

Der Kaffeebaum gelangte zu Anfang des 19. Jahrhunderts von Venezuela her, wo bereits 1784 bei Caracas Pflanzungen angelegt wurden, nach

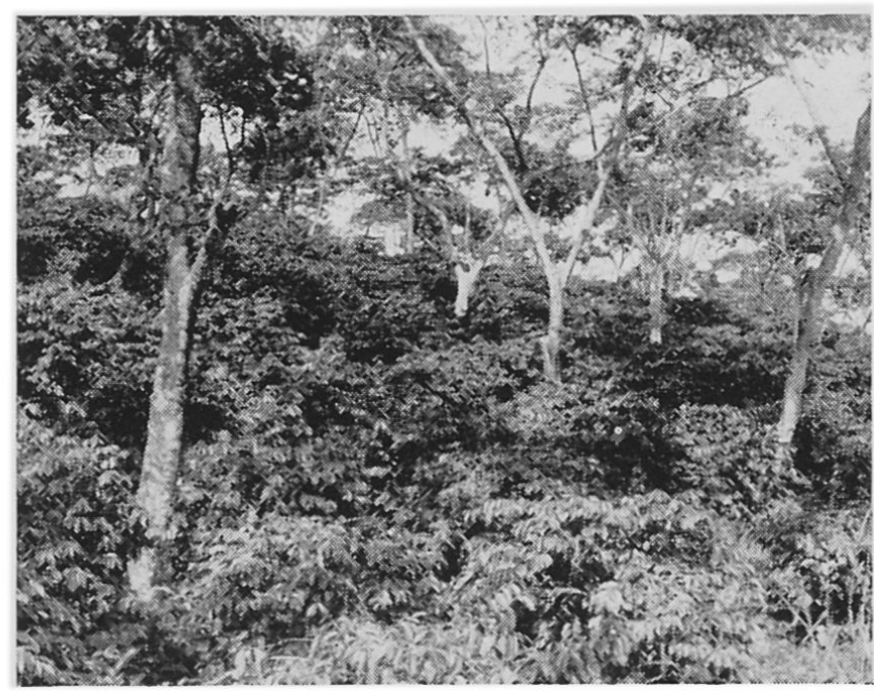

Abbildung 1. Kaffeepflanzung in typischer Hanglage. Die meist der Gattung Inga angehörenden Deck- oder Schattenbäume schützen die um $2 \mathrm{~m}$ hohen Kaffeebäumchen vor den Strahlen der grellen Tropensonne 
Kolumbien (7). Die ersten Pflanzungen in Kolumbien wurden im Grenzdepartement Santander angelegt, von wo sich der Anbau anscheinend zuerst in den Ostanden ausbreitete (3). Heute stammt der größte Teil der kolumbianischen Kaffeeproduktion (ungefähr $48 \%$ ) aus den Zentralanden. Auf die Westanden entfallen ungefähr $32 \%$, auf die Ostanden noch 18,5\%, die restlichen $1,5 \%$ wachsen an den Abhängen der Sierra Nevada de Santa Marta.

\section{Anbaugebiete und Anbaumethoden}

Die idealen Pflanzgebiete für den kolumbianischen Kaffee liegen an Berghängen, in Höhenlagen zwischen 1000 und $2000 \mathrm{~m}$, in der Zone der «tierra templada». Vereinzelte Kaffeepflanzungen werden allerdings auch in niedrigeren Höhenlagen angelegt. Kolumbien zerfällt dank seiner reichen Vertikalgliederung klimatisch in mehrere sehr verschiedenartige Provinzen. Daneben besitzt das Land aber auch eine klimatische Gliederung in horizontaler Richtung, wobei alle Gebiete unter $1000 \mathrm{~m}$ zum heißen Land (Tierra caliente), von 1000 bis $2000 \mathrm{~m}$ zum gemäßigten Land (Tierra templada) mit Durchschnittstemperaturen von $20-23^{\circ}$, von 2000 bis $3000 \mathrm{~m}$ zum kalten Land (Tierra fría), mit $12-16^{\circ}$, und von $3000 \mathrm{~m}$ bis zur Schneegrenze (rund $4700 \mathrm{~m}$ ) zum gefrorenen Land (paramos de la Tierra helada) gehören.

Als Beispiel für einen repräsentativen Klimatyp der Kaffeezone sei das in den Zentralanden in $1360 \mathrm{~m}$ Höhe gelegene Chinchiná, wo sich eine Versuchsanstalt der Federación Nacional de Cafeteros befindet, erwähnt. In den Jahren 1952-1955 $(5,6)$ betrugen bei einer Durchschnittstemperatur von $21,4^{\circ}$ die Maximaltemperaturen (im Monatsmittel) $32,0^{\circ}$ (März 1952) und die Minimaltemperatur $10,8^{\circ}$ (Juni 1955). Die durchschnittliche Niederschlagsmenge betrug $2822 \mathrm{~mm}$ im Jahr. Niederschläge wurden in allen Monaten verzeichnet (durchschnittliche Zahl der Regentage im Jahr 267), mit Niederschlagsminima in den Monaten Januar, Februar, März und August. Niederschlagsärmster Monat war der August 1953, als es in 10 Tagen $59,3 \mathrm{~mm}$ regnete; am niederschlagsreichsten war der Dezember 1955 mit 421,6 mm und 27 Regentagen.

Die in Kolumbien angebauten Kaffeearten sind Varietäten der ursprünglich aus Nordostafrika stammenden Art Coffea arabica. Zwar beschreibt Perez Arbalez (3) eine einheimische Art, Coffea sumapacénsis, die 1932 in den Ostanden entdeckt wurde; doch wird unseres Wissens dieser Kaffee nicht kultiviert.

Bis in die jüngste Vergangenheit wurden auf den kolumbianischen Kaffeehaziendas die Kaffeebäum-

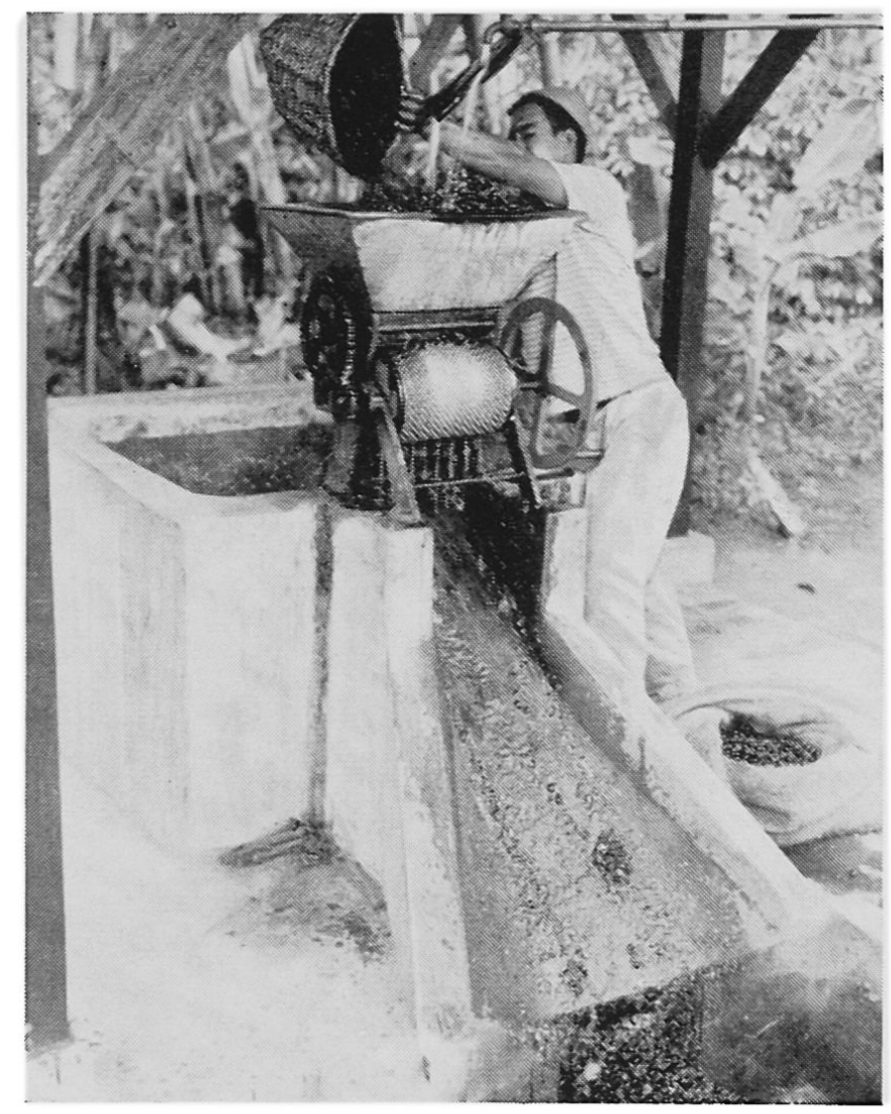

Abbildung 2. Despulpadora. Mit von Hand getriebenen Enthülsungsmaschinen werden die Kaffeebohnen von der fleischigen Umhüllung, der Pulpa, befreit

chen ausschließlich im Schatten von Deckbäumen kultiviert, die die Kaffeepflanzen vor der grellen Tropensonne schützen. Auf jungen Kaffeepflanzungen nehmen oft Bananen- und Platanopflanzen den Platz von Deckbäumen ein, bis diese zu ihrer vollen schattenspendenden Größe herangewachsen sind. Diese Deckbaumbestände geben der Landschaft der Kaffeezone ein ausgesprochenes Gepräge.

Am verbreitesten unter den Deckbäumen sind verschiedene Varietäten der zu den Leguminosen gehörenden Gattung Inga, 6 bis $10 \mathrm{~m}$ hohe Bäume, die in Kolumbien Guamo genannt werden. Die bekanntesten sind Guamo santafereño (Inga edulis), Guamo machete (Inga spectabilis) und Guamo rosario (Inga nobilis (3).

Die Deckbäume schirmen aber nicht nur die Sonnenstrahlen $a b$, sie halten mit ihren Wurzeln auch das Erdreich zusammen und vermindern die Bodenerosion, die bei den starken tropischen Regenfällen an den oft an steilen Hängen gelegenen Kaffeepflanzungen sonst katastrophale Ausmaße annehmen würde.

In den letzten Jahren ist in Kolumbien eine milde Arabicavarietät entwickelt worden, der sogenannte Caturra, der ohne Schattenbäume kultiviert wird. Caturrabäumchen sind niedriger und deshalb leich- 
ter zu pflegen und zu ernten als die übrigen in Kolumbien gepflanzten Kaffeebäume. Da sich mehr Caturrabäumchen je Flächeneinheit kultivieren lassen, werden um ungefähr $20 \%$ vergrößerte Hektarerträge erzielt. Da aber erst wenige Kaffeepflanzungen mit Caturrabäumchen bestockt sind, sind vollwertige Vergleichszahlen noch nicht erhältlich.

In den letzten sechs Kaffeejahren haben die Erträge je Flächeneinheit im Landesdurchschnitt leicht zugenommen, während die mit Kaffee bepflanzte Fläche abgenommen hat.

\begin{tabular}{|c|c|c|}
\hline 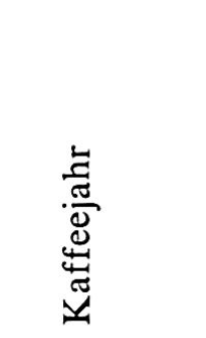 & 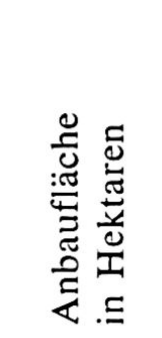 & 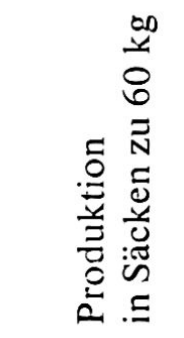 \\
\hline $1960 / 61$ & 831511 & 7500200 \\
\hline $1961 / 62$ & 824111 & 8035000 \\
\hline $1962 / 63$ & 810007 & 7580000 \\
\hline $1963 / 64$ & 813100 & 7800000 \\
\hline $1964 / 65$ & 812000 & 7900000 \\
\hline $\begin{array}{l}1965 / 66 \\
\text { (Schätzung) }\end{array}$ & 811400 & 7600000 \\
\hline
\end{tabular}

Die Hektarerträge schwanken allerdings von Produktionszone $\mathrm{zu}$ Produktionszone beträchtlich.

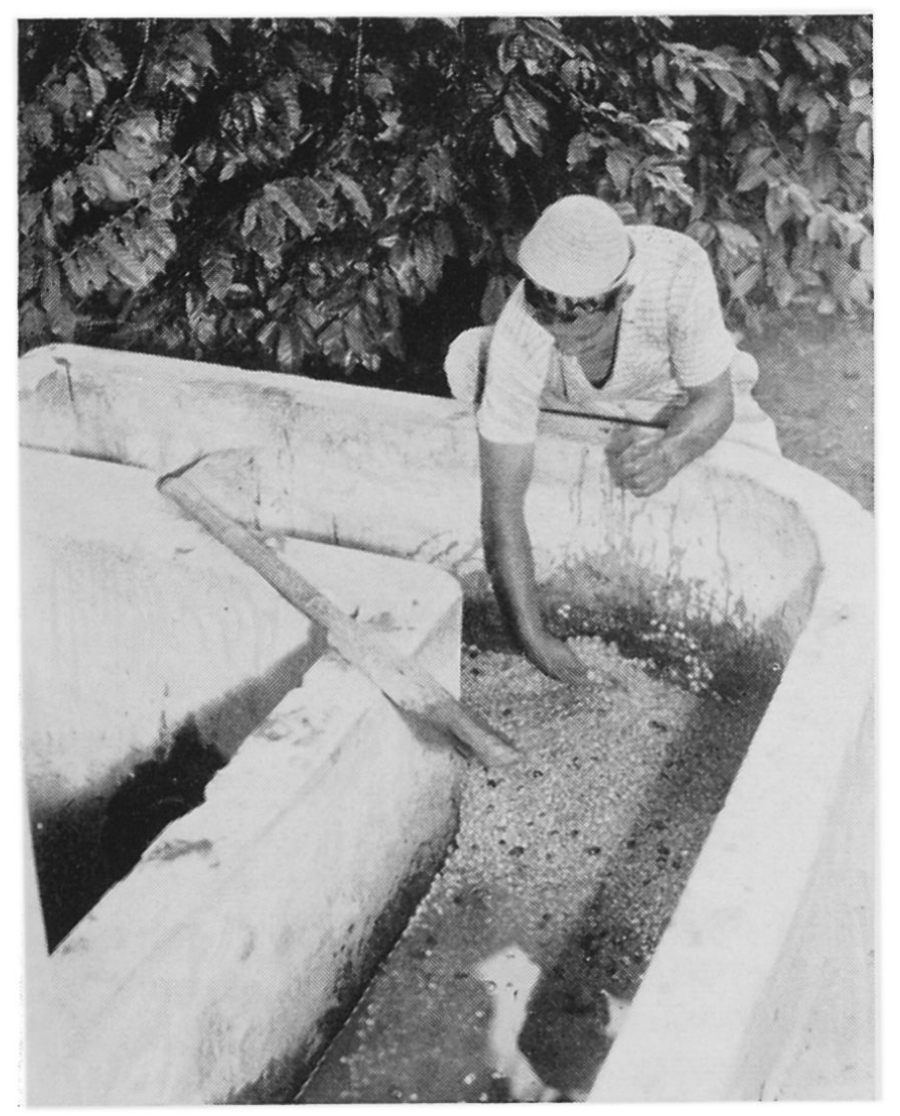

Abbildung 3. Im Wassertank werden die enthülsten Kaffeebohnen etwa einen Tag lang gewaschen
Während sie im eigentlichen Herzen der Kaffeezone, im ehemaligen Departement Caldas, das heute in die drei Departemente Caldas, Riseralda und Quindío aufgeteilt ist, gegen $700 \mathrm{~kg} / \mathrm{ha}$ betragen, werden in Nariño, dem südlichsten in den Anden gelegenen Departement des Landes, im Durchschnitt nur $200 \mathrm{~kg} / \mathrm{ha}$ geerntet.

Natürlich hängen die Erträge stark von der Witterung ab. Ein anderer Faktor, der den Ertrag der Pflanzungen wesentlich beeinflußt, ist das Alter der Kaffeebäumchen. Eine neuangelegte Kaffeepflanzung bringt die ersten Erträge nach 2 Jahren; Vollernten werden aber erst nach 4-5 Jahren erzielt; über 15 Jahre alte Kaffeebäume gehen im Ertrag stark zurück. 1963, dem letzten Jahr, von dem vollständige Zahlen vorliegen, betrug die Zahl der Kaffeebäume in Kolumbien 2025 Millionen. Davon waren 219 Millionen noch unproduktive Jungpflanzen. 1963 war der größte Teil der produzierenden Bäume über 15 Jahre alt.

$\begin{array}{cc}\text { Alter (in Jahren) } & \% \text { der Gesamtzahl } \\ \text { unter } 4 & 8,2 \\ 4-9 & 18,5 \\ 10-15 & 13,7 \\ \text { über } 15 & 59,6\end{array}$

1956, einem anderen $\mathrm{Jahr}$, von dem vollständige Zahlenangaben vorliegen, war der Altersaufbau der kolumbianischen Kaffeepflanzungen und die Produktivität der verschiedenen Altersstufen der folgende:

\begin{tabular}{|c|c|c|}
\hline 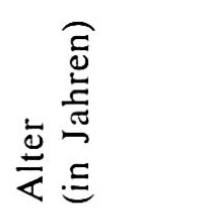 & 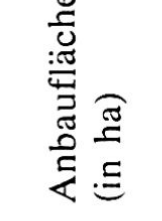 & 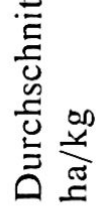 \\
\hline 1 & 21601 & - \\
\hline 2 & 26603 & 31 \\
\hline 3 & 34361 & 187 \\
\hline $4-6$ & 60938 & 463 \\
\hline $7-9$ & 51825 & 556 \\
\hline $10-12$ & 76482 & 580 \\
\hline $13-15$ & 65998 & 542 \\
\hline älter als 15 & 430995 & 514 \\
\hline
\end{tabular}

Der kolumbianische Kaffee wird nur zu einem kleinen Teil auf Großhaziendas produziert. Der größte Teil der Kaffeepflanzungen sind Kleinbetriebe (Fincas), meist Familienbetriebe, wo die Arbeiten vom Pflanzer und seinen meist zahlreichen Familiengliedern besorgt wird und nur zur Erntezeit fremde Hilfskräfte beigezogen werden. 
Im Jahre 1956 ergab sich folgende Besitzverteilung:

\begin{tabular}{|c|c|c|c|c|}
\hline 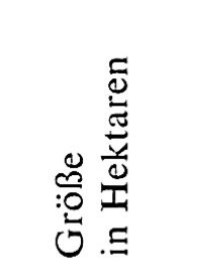 & 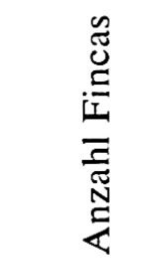 & $\%$ & 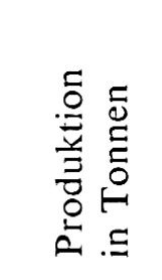 & $\%$ \\
\hline unter 1 & 77245 & 36,3 & 19129 & 5,3 \\
\hline $1,1-10$ & 123719 & 58,1 & 207639 & 57,9 \\
\hline $10,1-50$ & 11429 & 5,4 & 108637 & 30,3 \\
\hline $50,1-100$ & 447 & 0,2 & 13734 & 3,9 \\
\hline $100,1-200$ & 79 & - & 4426 & 1,2 \\
\hline \multirow[t]{2}{*}{ über 200} & 51 & - & 4996 & 1,4 \\
\hline & 212970 & & 358561 & \\
\hline
\end{tabular}

1965 waren die Verhältnisse im wesentlichen gleich:

\begin{tabular}{lrr} 
Größe in Hektaren & Anzahl Fincas & \multicolumn{1}{c}{$\%$} \\
unter 1 & 109670 & 36,3 \\
$1,1-10$ & 178894 & 58,0 \\
$10,1-50$ & 16123 & 5,4 \\
über 50 & 843 & 0,3 \\
\hline
\end{tabular}

301530

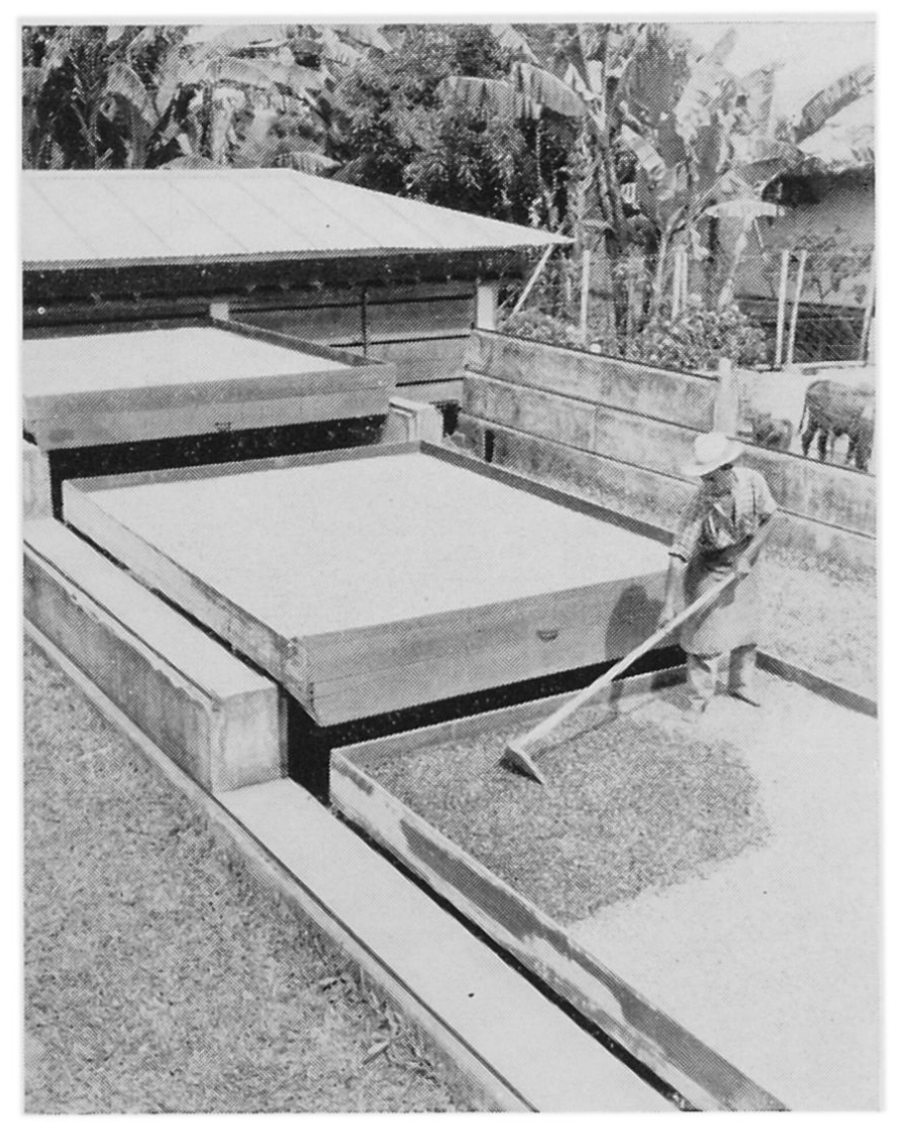

Abbildung 4. Nach der Waschung wird der Kaffee an der Sonne getrocknet. Durch mehrmaliges Umwälzen mit hölzernen Schaufeln werden die Bohnen von allen Seiten der Sonne ausgesetzt
$62,3 \%$ der Produktion stammte 1965 von Betrieben mit weniger als 10 ha Produktionsfläche. Die große Zahl von Kleinbetrieben machen die an und für sich - angesichts der chronischen weltweiten Überproduktion von Kaffee - etwas eigentümlich anmutenden Bemühungen um die Steigerung der Hektarerträge durch Einführung der Caturra-Varietät leichter verständlich. Die für diese Politik Verantwortlichen hoffen, dadurch den Kleinpflanzern die Möglichkeit zu geben, die mit Kaffee bepflanzte Fläche einzuschränken und auf den so gewonnenen Flächen andere Produkte anzubauen, um so mindestens die Selbstversorgung der Kaffeepflanzer mit landwirtschaftlichen Produkten zu erreichen oder wenigstens zu verbessern.

So hofft man, im Laufe von fünf Jahren die typische auf Monokultur eingestellte Kaffeehazienda in einen gemischten Landwirtschaftsbetrieb zu verwandeln.

Die dominierende Stellung des Kaffeeanbaus im Rahmen der kolumbianischen Landwirtschaftsproduktion wird durch folgende Zusammenstellung der Anbauflächen und Ernteschätzungen aus dem Jahre 1966 unterstrichen (Viehwirtschaft nicht berücksichtigt).

\begin{tabular}{|c|c|c|c|c|}
\hline & 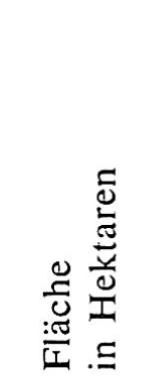 & $\begin{array}{l}\circ^{\circ} \\
. \Xi\end{array}$ & 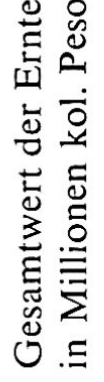 & $\begin{array}{l}\text { ○ }^{\circ} . \Xi\end{array}$ \\
\hline Kaffee & 811000 & 24,8 & 3200 & 28,9 \\
\hline Zuckerrohr & 82000 & 2,5 & 1286 & 11,6 \\
\hline Platanos & 330000 & 10,1 & 1120 & 10,1 \\
\hline Reis & 376000 & 11,5 & 980 & 9,0 \\
\hline Mais & 700000 & 21,4 & 815 & 7,4 \\
\hline Baumwolle & 176000 & 5,4 & 645 & 5,9 \\
\hline Yuca & 220000 & 6,7 & 638 & 5,8 \\
\hline Kartoffeln & 82000 & 2,5 & 590 & 5,3 \\
\hline Weizen & 102000 & 3,1 & 307 & 2,9 \\
\hline Bananen & 32000 & 1,0 & 292 & 2,8 \\
\hline Andere & 360000 & 11,0 & 1138 & 10,3 \\
\hline
\end{tabular}

Die Kaffeepflanzer ziehen sich ihre jungen Kaffeepflanzen im allgemeinen selber in kleinen Pflanzgärten, aus denen die Schößlinge, wenn sie etwa $50 \mathrm{~cm}$ hoch sind, in die eigentliche Kaffeepflanzung versetzt werden; dort wachsen sie im Schatten der Deckbäume, die nicht nur die grellen Sonnenstrahlen abhalten, sondern auch zu einer gleichmäßigen Luftfeuchtigkeit und Wärme beitragen, rasch weiter. Sie werden allerdings daran gehindert, ihre volle Höhe von $5-7 \mathrm{~m} \mathrm{zu}$ erreichen, und auf die für Ernte und Pflege günstige Höhe von etwa $2 \mathrm{~m}$ zurückgeschnitten. Die Bäumchen der neuen Varietät 

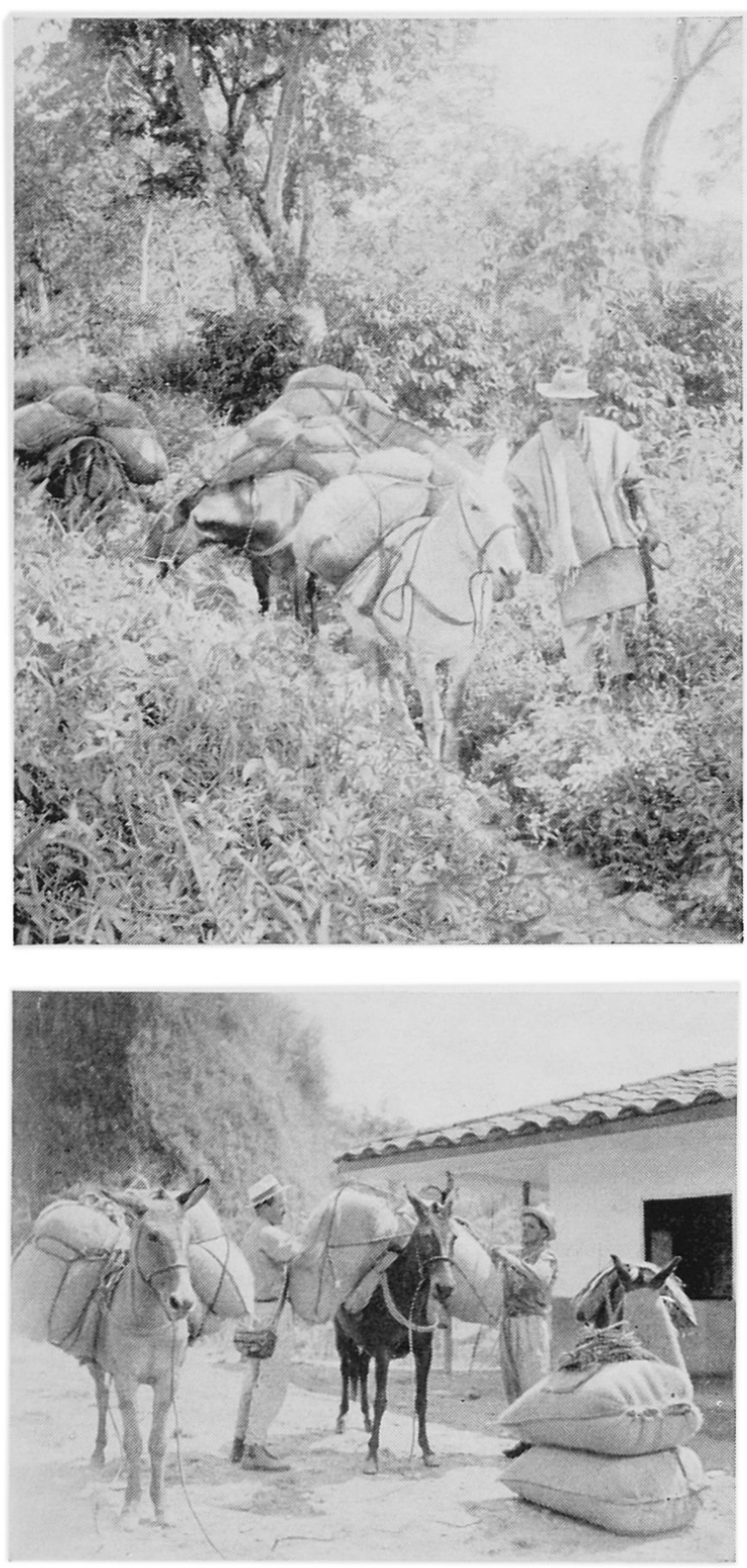

Abbildungen 5/6. Der Transport von der Kaffeefinca zur Abnahmestelle der Federación de Cafeteros erfolgt wegen dem unwegsamen Gelände meist auf dem Rücken von Maultieren

Caturra erreichen im ausgewachsenen Zustand ungefähr diese Höhe.

Normalerweise dauert der Zyklus von der weißen Blüte bis zur ausgereiften Frucht ungefähr sieben Monate. Auf einer Fahrt durch die Kaffeezone, etwa auf einer der Straßen, die Bogotá mit dem Magdalenental verbindet, sieht man aber immer wieder auf dem gleichen Bäumchen neben Blüten grüne, unausgereifte Beeren und die roten vollausgereiften Früchte, die wie kleine Kirschen aussehen. Da nur vollausgereifte Früchte geerntet wer- den, zieht sich die Kaffeernte über Wochen hin, da die Pflücker immer wieder zu den gleichen Bäumchen zurückkehren müssen, um später ausgereifte Beeren zu lesen.

Damit die Kaffeebohne transportfähig wird, muß sie von der fleischigen Umhüllung, der Pulpa, befreit werden, was meist auf der Hazienda auf maschinellem Wege, allerdings in der Großzahl der Betriebe mit kleinen, handgetriebenen Enthülsungsmaschinen (despulpadoras) geschieht. Die Kaffeebohnen, die je zu zweit von einem Häutchen, dem Pergamino, eingeschlossen sind, gelangen nun in einen Wassertank, wo sie etwa einen Tag lang bleiben. Nachher werden sie an der Sonne getrocknet - wiederum eine Stufe der Kaffeeaufbereitung, die jedem Reisenden durch Kolumbien auffällt. In speziellen Trocknungströgen auf großen Haziendas, auf Zementplattformen neben den kleinen Kaffeefincas, aber auch auf der Straße vor den Häusern und auf dem Dorfplatz der Kaffeezone werden die Bohnen durch konstantes Umwälzen mit einer hölzernen Schaufel von allen Seiten der Sonne ausgesetzt. Das getrocknete Produkt, der «pergamino», wird meist von kleineren Produzenten an die durch die gesamte Kaffeezone verteilten Ablieferungsstellen der Federación Nacional de Cafeteros oder auch direkt an die privaten Exporteure verkauft.

Der Transport von der Kaffeefinca zur Ablieferungsstelle erfolgt wegen des gebirgigen Terrains immer noch zu überwiegendem Teil mit Maultieren. Die Inlandankaufspreise werden denn auch meist in "cargas», Maultierlasten zu $125 \mathrm{~kg}$, festgelegt. Eine «carga» Kaffee zerfällt in 2 Säcke zu $62,5 \mathrm{~kg}$. Kolumbianische Kaffeeausfuhren werden normalerweise nicht in Tonnen, sondern in der Anzahl der ausgeführten Säcke in den Statistiken genannt. Die für die Ausfuhr verwendeten Säcke enthalten aber nur $60 \mathrm{~kg}$ Kaffee. Diese Verschiedenheit der Sackgewichte ist für den ausländischen Beobachter verwirrend und führt immer wieder zu anscheinenden Unstimmigkeiten zwischen Produktions- und Ausfuhrstatistiken.

Vor der Ausfuhr werden die Kaffeebohnen in der "Trilladora» von ihren umhüllenden Häutchen befreit und einer scharfen Sortierung nach Größe, Gewicht und Form unterzogen. Bevor der Kaffee schließlich zum Export zugelassen wird, unterliegt er einer neuerlichen Qualitätskontrolle durch Experten der Federación de Cafeteros, damit nur erstklassiger kolumbianischer Kaffee auf den Weltmarkt gelangt.

\section{Die Federación Nacional de Cafeteros de Colombia}

Die Federación Nacional de Cafeteros de Colombia ist eine halbstaatliche "non profit»-Organisation. Sie wurde im Jahre 1927 gegründet, als Kolumbien 


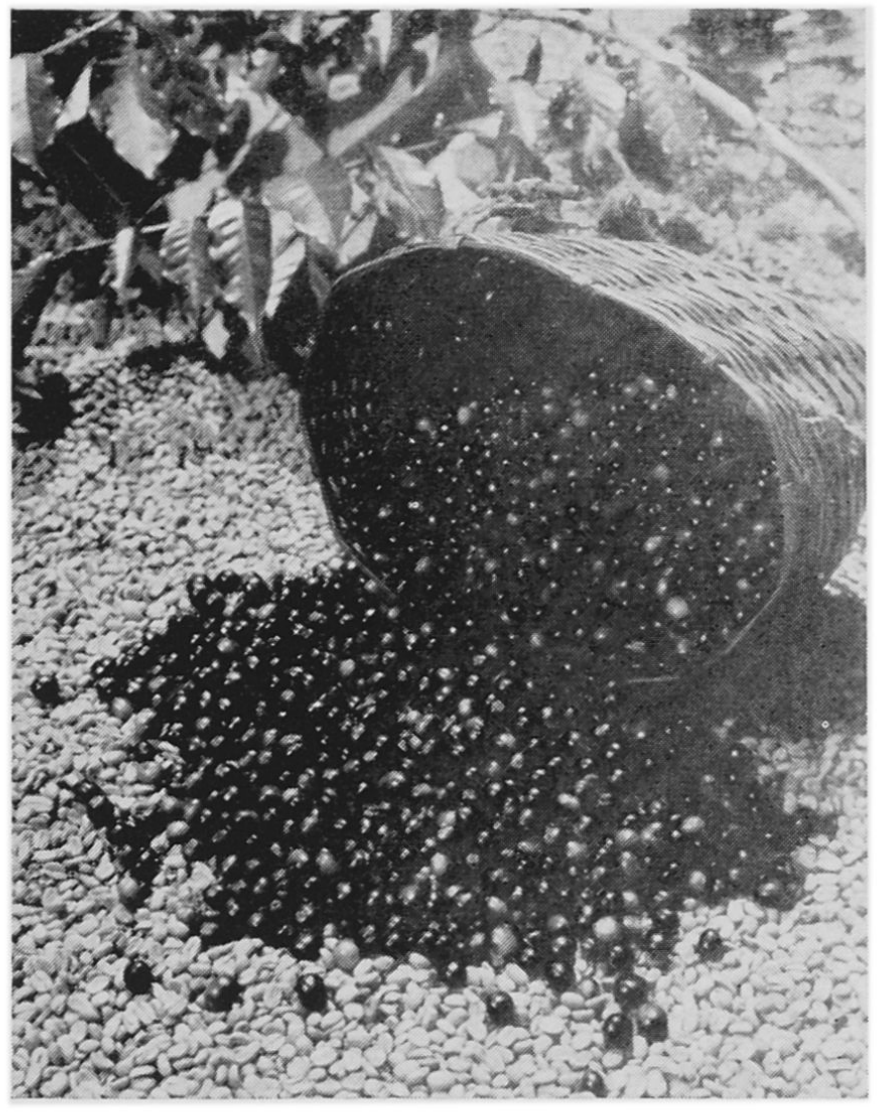

Abbildung 7. Frischgeerntete rote Kaffeebeeren exportbereiten Kaffeebohnen

Alle Aufnahmen: Federación Nacional de Cafeteros de Colombia liegen in scharfem Kontrast auf den enthülsten,

treut die Federación aber auch das kaffeebauliche landwirtschaftliche Versuchswesen und leistet einen erheblichen Beitrag zur Erforschung des kolumbianischen Lokalklimas, indem sie ein Netz von über 20 regelmäßig bedienten meteorologischen Stationen aufgebaut hat. Durch Wegebau, Anleitungen zum zweckmäßigen Hausbau, Anweisungen für fortschrittliche Produktionsmethoden, Unterstützung des Anbaus von zusätzlichen landwirtschaftlichen Produkten, einerseits um die Kaffeepflanzer der Selbstversorgung näherzubringen, andererseits um ihnen zusätzliche Einnahmequellen zu erschließen und sie dadurch zur Einschränkung der Kaffeeproduktion zu stimulieren, sucht die Federación den Lebensstandard der kolumbianischen Kaffeepflanzer zu heben und die Basis der kolumbianischen Volkswirtschaft zu erweitern.

Durch diese vielfältigen Funktionen ist die Federación seit Jahren der wichtigste Einzelfaktor in der kolumbianischen Volkswirtschaft. Die Marktabschöpfung in einem Markt mit fallenden Preisen und die vielen anderen Maßnahmen, die die Federación zu Gunsten der Cafeteros unternimmt, haben allerdings im Laufe der Jahre zu einer enormen Verschuldung geführt.

Ende März 1967 betrug die Verschuldung des Nationalfonds der Kaffeeproduzenten 2,5 Milliarden Pesos (über 750 Millionen Fr.).

\section{Die kolumbianische Kaffeeausfuhr}

mit damals 2,3 Millionen Säcken Kaffee langsam zu einem wichtigen Faktor auf dem Weltmarkt heranwuchs. Die Federación legt Produktionsstandards und Minimalinlandankaufspreise für den kolumbianischen Kaffee fest und kauft, wenn notwendig, zu diesen Preisen den Kaffee direkt beim Produzenten ein, um ihn bei gedrückter Marktlage einzulagern und später nach Möglichkeit zu exportieren. Sie besorgt die Propaganda für den kolumbianischen Kaffee im Ausland, schaltet sich in internationale Kompensationsgeschäfte ein und hat maßgeblichen Anteil an den offiziellen Vertretungen Kolumbiens an den Weltkaffeekonferenzen. Durch den von ihr gegründeten Banco de Cafetero, an dessen Aktienkapital sie mehrheitlich beteiligt ist, finanziert sie neue Kaffeepflanzungen und bevorschußt Ernten. Sie ist der kolumbianische Hauptaktionär der Flota Mercante Gran Colombiana S.A., an deren Gründung sie entscheidend beteiligt war (10). Im letzten Jahr wurden auf Schiffen dieser kolumbianisch-ekuadorianischen Schiffahrtsgesellschaft - die venezolanischen Partner sind anfangs der fünfziger Jahre ausgeschieden - 35\% des kolumbianischen Kaffees exportiert.

Neben diesen rein wirtschaftlichen Funktionen be-
Der größte Teil der kolumbianische Kaffeexporte erfolgt durch den Hafen von Buenaventura (84\% in den Jahren 1956-1960, 80\% in den Jahren 1961-1965). In weitem Abstand folgen die Häfen am Karibischen Meer (Cartagena 8, bzw. 13\% der Kaffeeausfuhren, Barranquilla 7 bzw. 2,5\% und Santa Marta 0,3 bzw. 4\%). Der Rest der Ausfuhren erfolgt über den Pazifikhafen Tumaco und über Cucuta. Nicht erfaßt in den Ausfuhrstatistiken werden allerdings die zeitweise sehr beträchtlichen Mengen von geschmuggeltem Kaffee, die namentlich über die kolumbianisch-venezolanische Grenze außer Landes geschafft werden.

In der Verlagerung der Ausfuhr von Barranquilla nach Santa Marta seit dem Jahre 1961 spiegelt sich die Inbetriebnahme der Magdalenatalbahn von La Dorada nach Santa Marta wider, die ein beträchtliches Frachtvolumen von der Magdalenaflußschifffahrt, die in Barranquilla endet, abgezogen hat.

Traditionell sind die Vereinigten Staaten von Amerika bei weitem die größten Importeure von kolumbianischem Kaffee, wobei allerdings in den letzten Jahren der afrikanische Kaffee zum großen Teil auf Kosten der Einfuhren aus Kolumbien seinen Marktanteil in den USA vergrößert hat. Dafür haben sich 
im Laufe der zehnjährigen Vergleichsperiode die Ausfuhren nach Europa stark vergrößert.

Wichtigste Abnehmerländer

von kolumbianischen Kaffee

in Säcken zu 60 kg 1956

1965

\begin{tabular}{lrr} 
USA & 4258834 & 3048905 \\
Europa & 651064 & 2399986 \\
davon Westdeutschland & 259247 & 807981 \\
\multicolumn{1}{c}{ Schweden } & 128080 & 302120 \\
\cline { 2 - 3 } Gesamtausfuhren & 5069777 & 5651544
\end{tabular}

\section{Literatur}

1 Foreign Agriculture Circular FCOF, 2-66.

2 Banco de la Republica. Depto. Investigaciones Economicas 1966.

3 Enrique Perez Arbelaez: Plantas utiles de Colombia. Contraloria General de la Republica, Bogotá 1947.

4 Boletin de Información Estadistica sobre cafe, Federación Nacional de Cafeteros, Asesoría de Investigaciones Economicas, No. 40, 1966.

5 Anuario Meteorologico 1952, 1953, 1954. Min. de Agricultura. Bogotá 1955.

6 Anuario Meteorologico 1955, Instituto Geografico "Agustin Codazzi». Bogotá 1961.

7 Marrero, Levi: Venezuela y sus recursos. Madrid 1964.
8 Convenio Internacional del Cafe 1962.

9 Revista del Banco de la Republica, No. 472. Bogotá, Febrero 1967.

10 Tanner Hans: Verkehrsprobleme Kolumbiens, Geogr. Helv. IV, 1949.

\section{Résumé}

Mis à part le Brésil, la Colombie est le pays producteur de café le plus important du monde. L'article de $M$. Tanner révèle le rôle que le café joue dans l'économie publique de ce pays. Il s'occupe d'abord des régions de la «tierra templada» (zone tempérée), située dans une altitude de 1000 à $2000 \mathrm{~m}$ et dans laquelle le café est cultivé, puis aux méthodes de plantation. Un autre chapitre intéressant est consacré à l'organisation de la vente et à l'exportation. On apprend à connaître, entre autres, les moyens qui permettront - comme on l'espère - de transférer le système de monocultures, caractéristique jusqu'à présent, dans un système d'exploitations mixtes fournissant d'autres produits agricoles encore. La Federación Nacional de Cafeteros de Colombia, fondée en 1927, représente l'organisme le plus important soit du point de vue de la création de nouvelles possibilités d'exportation soit de celui du financement des plantations, soit aussi de celui de l'exploration scientifique.
Nordmexiko liegt im Bereich der Trockenklimate und kann nur mit Hilfe künstlicher Bewässerung ackerbaulich genutzt werden. Das Vorhandensein von Wasser entscheidet über Leben und Tod, über eine ausreichende Maisernte oder Hungersnot. Der Mais, die Grundnahrung der Indios, genießt seit Jahrhunderten kultische und religiöse Verehrung, und bezeichnenderweise gehören die Regengötter, die noch heute an bäuerlichen Festen in Yucatán gefeiert werden, zu den wichtigsten altmexikanischen Gottheiten. Interessanterweise deckt sich ihre Erscheinung vielfach mit derjenigen christlicher Heiliger, z. B. Tlaloc, der Regengott der Tolteken, war so bedeutend, daß er von den Chichimeken und von den Azteken übernommen wurde.

Wasser benötigt die Mexikanerin aber auch zum
Waschen - denn peinliche Sauberkeit liebt oft gerade die Ärmste. Die einfache Frau wäscht ihr Baumwollkleid fast täglich, setzt sie sich doch ohne weiteres in ihrem weiten Rock auf den Boden und hält ihre Waren feil. Gewaschen wird in den Dörfern häufig in Gruppen am offenen Wasser, sei es an einer gemeinsamen Brunnenanlage, am fließenden oder stehenden Gewässer, mit oder ohne "Waschbrett-Einrichtung".

So vielgestaltig wie Mexiko in Sprache und Volkscharakter ist, so bunt wirkt die Kleidung der Frauen. Einerseits beeinflußt die altmexikanische Formenund Farbenpracht die heutige Mode - doch dokumentiert unser Bild eher den viel stärkeren europäischen Einfluß auf die Bekleidung der einheimischen Indianer und Mestizen. 
